\title{
Structural Properties of Complex (Dusty) Plasma upon Crystallization and Melting
}

\author{
B. A. Klumov ${ }^{a, b}$ and G. E. Morfill ${ }^{a}$ \\ ${ }^{a}$ Max Planck Institut für Extraterrestrische Physik, D-85740 Gaiching, Germany \\ ${ }^{b}$ Joint Institute for High Temperatures, Russian Academy of Sciences, \\ Izhorskaya ul. 13, Moscow, 125412 Russia
}

Received August 11, 2009

\begin{abstract}
A change in the local order of a bounded complex (dusty) plasma in the process of its crystallization and melting has been examined by molecular dynamics simulations. The dynamics of microparticles is considered in the framework of a Langevin thermostat, the pair interaction between charged particles is described by a screened Coulomb potential (Yukawa potential) with the hard wall potential as a confinement. It has been shown that the beginning of the crystallization of such a system is accompanied by the formation of clusters with the hexagonal close packed (hcp) structure; a noticeable number of these clusters are then transformed to the face centered cubic (fcc) phase. A plasma crystal formed after crystallization consists of the metastable hcp phase, fcc clusters, and a small number of clusters with a body centered cubic (bcc) crystal lattice. Beginning with a certain threshold value of the thermostat temperature, the number of fcc/bcc clusters decreases sharply with increasing temperature, which is an important signature of the beginning of the melting of the plasma crystal.
\end{abstract}

PACS numbers: 52.27.Lw, 61.20.Ja, 64.60.Cn

DOI: $10.1134 / \mathrm{S} 002136400918009 \mathrm{X}$

Interest in processes in a plasma containing microparticles has increased considerably in recent years. Such a plasma is usually called a complex plasma or dusty plasma (see, e.g., [1, 2]). First, complex plasma is of interest due to its great abundance in nature. Interstellar clouds, gas-dust clusters, planetary rings [3], comet atmospheres [4], and the ionospheres and magnetospheres of planets are complex plasmas to a certain extent, where dust particles often decisively affect the state of the system containing them. For example, noctilucent clouds [5] formed in the cold dusty upper Earth's atmosphere are dusty structures determining the ionization properties of the mesosphere. Dusty ejecta formed in the high-speed collision between celestial bodies of the solar system provide important information on the chemical composition of the projectile and target [6], etc. Second, modern laboratory experiments make it possible to trace the behavior of a single microparticle providing the most detailed kinetic description of the properties of the ensemble of dust particles. Owing to these circumstances, dusty plasma is an attractive tool for studying various fundamental physical problems such as phase transitions [7, 8], hydrodynamic instabilities [9], crystallization waves [10], etc. One of these important problems is a change in the local order of the dust component of complex plasma in the process of its crystallization and melting, which is discussed in this work. Note that it is technically possible now to determine the $x, y$, and $z$ coordinates of $N \sim 10^{5}$ micropar- ticles in a characteristic time of $\tau_{s} \simeq 1-10 \mathrm{~s}$ [11-13], which for the time being is larger than the typical times of the indicated phase transitions in the complex plasma. A decrease in $\tau_{s}$ by an order of magnitude will allow for an experimental investigation of the kinetics of the melting and crystallization of the complex plasma; such an advance is expected in the near future.

Under laboratory conditions, a complex (dusty) plasma is usually obtained by introducing microparticles into a weakly ionized low-temperature gas-discharge plasma of low-pressure inert gases. The recombination of electrons and ions on the surface of dust particles gives rise to the fast charging of the particles; the charge value depends on the size of a particle and the plasma parameters; for example, a $1-\mu \mathrm{m}$ particle in a usual microwave discharge in argon acquires a negative charge of $Z_{\mathrm{d}} \sim 10^{3} e$, where $e$ is the elementary charge. Such a large charge of the microparticle often results in the strong nonideality of the dust component, which can be in various phase states, i.e., can be manifested as a gas, liquid, or crystal. The crystal state of the dust component of the complex plasma (plasma crystal) was experimentally discovered in 1994 [14, 15], being theoretically predicted in 1986 [16].

Owing to the fast diffusion of electrons towards the walls of the discharge chamber, the central region of the gas discharge is positively charged and is a potential well (confinement) for negatively charged microparticles. The profile of the confining potential in the 
central region of the discharge is close to a parabolic one [17]. The electric field in the near-electrode region of the discharge increases much more strongly than that in the center and the confinement in this case can be described as a hard wall potential.

The main aim of this work is to analyze a change in the local properties of the three-dimensional complex plasma in the process of its crystallization/melting. For simplicity, the pair interaction between microparticles in complex plasma is described by a screened Coulomb potential (Debye-Hückel or Yukawa potential) ${ }^{1}$

$$
\phi(r)=\left(Z_{\mathrm{d}} / r\right) \exp \left(-r / \lambda_{\mathrm{D}}\right),
$$

where $r$ is the distance between the particles and $\lambda_{\mathrm{D}}$ is the characteristic screening length. Since we are interested in the features of crystallization/melting, the complex plasma under consideration is strongly nonideal. This means that the coupling parameter of the indicated dust subsystem is large,

$$
\Gamma \equiv \frac{Z_{\mathrm{d}}^{2}}{T_{\mathrm{d}} \Delta} \exp (-\kappa) \gg 1,
$$

where $T_{\mathrm{d}}$ is the temperature of the microparticles, $\Delta$ is the mean distance between the particles, and $\kappa=\Delta / \lambda_{\mathrm{D}}$ is the structure parameter. The behavior of the ensemble of microparticles is investigated by the molecular dynamics simulations. For simplicity, it is accepted that all of the microparticles have a fixed size of $a \approx$ $1 \mu \mathrm{m}$ and a fixed charge of $Z_{\mathrm{d}} \sim 3 \times 10^{3} e$. The equations of motion of an individual microparticle have the form

$$
m \ddot{\mathbf{r}}_{i}=-Z_{\mathrm{d}} \nabla \Phi_{\mathrm{c}}-Z_{\mathrm{d}} \sum \nabla \phi-m \gamma \dot{\mathbf{r}}_{i}+\mathbf{L}_{i},
$$

where the terms on the right-hand side describe the electrostatic interaction between the particles; the drag of the microparticles due to collisions with neutral atoms and molecules of the buffer gas; the random Langevin force $\mathbf{L}_{i}$ (thermal noise induced by neutral particles), which is determined from the equation $\left\langle\mathbf{L}_{i}(t) \mathbf{L}_{j}(t+\tau)\right\rangle=2 \gamma m k_{\mathrm{B}} \delta_{i j} \delta(\tau)$ under the condition of zero mean value $\left\langle\mathbf{L}_{i}(t)\right\rangle=0$; and the interaction of the microparticles with the confining potential $\Phi_{\mathrm{c}}$ ). The system of Eqs. (3) describes the so-called Langevin thermostat, because the temperatures of the gas and dust particles are equal to each other at large times.

System (3) was solved for $N=8000$ microparticles randomly distributed in a cube with a size $L$ at the initial time. The confinement bounds the position of the microparticles along the $z$ axis $(-L / 2 \leq z \leq L / 2)$. We used periodic boundary conditions at the lateral edges of the cube. The confining electric field increases exponentially at $|z|>L / 2$ with the typical spatial scale

\footnotetext{
${ }^{1}$ The particle interaction potential in complex plasma was measured in [17] and appeared to be close to a Yukawa potential.
}

$d_{\mathrm{w}} \simeq \Delta / 3$, preventing microparticles from going away from the calculation region by distances $l>d_{\mathrm{w}}$. The other simulation parameters were taken to be close to the real experimental parameters: $\kappa \simeq 1-3$; buffer gas pressure and temperature $p_{\mathrm{g}} \approx 10-100 \mathrm{~Pa}$ and $T_{\mathrm{g}} \sim$ 100-1000 K, respectively; and microparticle density $\rho_{\mathrm{d}} \approx 1.4 \mathrm{~g} / \mathrm{cm}^{3}$. Owing to friction, such a system at large times (when $T_{\mathrm{d}} \approx T_{\mathrm{g}}$ ) is crystallized with the coupling parameter $\Gamma \sim 10^{3}-10^{5}$.

To determine the local order in the system of microparticles, it is convenient to use the method of local rotational invariants [18]. In the framework of this approach, $N_{b}(i)$ nearest neighbors are first determined for each $i$ th particle by methods of Delone simplexes and Voronoi cells. The number $N_{b}$ is usually larger than $N_{m} \simeq \frac{4}{3} \pi r_{m}^{3} \frac{N}{V}$, where $V=L^{3}$ is the volume occupied by microparticles, $N$ is the total number of the particles in the system, and $r_{m}$ is the distance to the first minimum of the pair correlation function $g(r)$ given by the expression

$$
g(r)=\frac{L^{3}}{N^{2}}\left\langle\sum_{i}^{N} \sum_{j \neq i} \delta\left(\mathbf{r}-\mathbf{r}_{i j}\right)\right\rangle .
$$

The local orientational order $q_{l m}(i)$ for each microparticle can be defined in terms of the vectors $\mathbf{r}_{i j}$ connecting the $i$ th particle with the nearest neighbors $(j=1$, ..., $N_{b}$ ) as

$$
q_{l m}(i)=\frac{1}{N_{\mathrm{b}}(i)} \sum_{j=1}^{N_{b}(i)} Y_{l m}\left(\theta_{j}, \phi_{j}\right),
$$

where $Y_{l m}(\theta, \phi)$ are the spherical harmonics and $\theta_{j}$ and $\varphi_{j}$ are the angular coordinates of the $j$ th particle specified by the vector $\mathbf{r}_{i j}$. Using $q_{l m}(i)$ values, which depend on the choice of the coordinate system (and, thus, are of little use to determine the local orientational order), the second-order rotational invariants $q_{l}(i)$ can be calculated for every microparticle by the formula

$$
q_{l}(i)=\left(\frac{4 \pi}{(2 l+1)} \sum_{m=-l}^{m=l}\left|q_{l m}(i)\right|^{2}\right)^{1 / 2} .
$$

It is worth noting that each crystal lattice type has its individual set of $q_{l}$. This makes it possible to identify the observed crystal structure by comparing the calculated $q_{l}$ values for each microparticle with the $q_{l}^{\text {id }}$ values for ideal lattices.

To identify the crystal structure, the second-order rotational invariants $q_{4}$ and $q_{6}$ are usually used; they are easily calculated for the perfect fcc/hcp/ico/bcc crystals (see, e.g., $[18,19]$ ). For the fcc/hcp/ico clusters, the number of nearest neighbors of each particle 

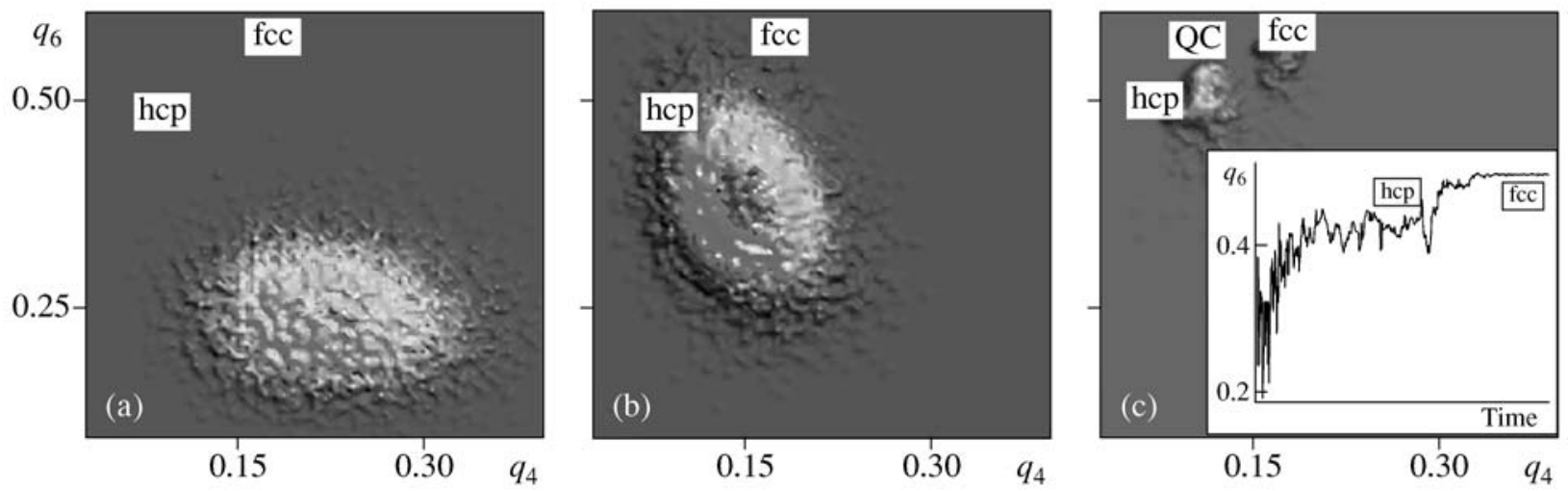

Fig. 1. (Color online) The distribution of microparticles on the $q_{4}-q_{6}$ rotational invariant plane, which demonstrates the structural properties of the confined Yukawa system at various crystallization stages: (a) the initial uncorrelated state of the ensemble of particles, (b) the glassy state of most of the particles and the appearance of the hcp-like clusters as precursors of crystallization, and (c) the almost complete crystallization of the system of microparticles. The inset shows the time evolution of the parameter $q_{6}$ for a typical fcc-like microparticle. The rotational invariants $q_{4}$ and $q_{6}$ are calculated with $N_{b}=12$.

is $N_{b}=12$ and these invariants are $q_{4}^{\mathrm{fcc}}=0.1909$ and $q_{6}^{\mathrm{fcc}}=0.5745$ for the fcc lattice, $q_{4}^{\mathrm{hcp}}=0.0972$ and $q_{6}^{\text {hcp }}=0.4847$ for the hcp phase, and $q_{4}^{\text {ico }}=0$ and $q_{6}^{\text {ico }}=0.6633$ for the icosahedral (ico) phase.

Owing to large $q_{6}^{\text {cr }}$ values for all of the above types of crystal lattices, the initial stages of nucleation/crystallization can be examined for various systems [20]. Note that the mean value for a weakly correlated system of particles (gas/liquid) is $\left\langle q_{6}\right\rangle \simeq N_{b}^{-\frac{1}{2}}$ and is much smaller than $q_{6}^{\text {cr }}$; for example, $\left\langle q_{6}\right\rangle \approx 0.29$ for $N_{b}=12$.

For the bcc lattice and $N_{b}=8$ (i.e., when the crystal lattice under consideration is simple cubic), $q_{4}^{\mathrm{sc}}=$ 0.5092 and $q_{6}^{\text {sc }}=0.6285$. To determine the bcc clusters, the position of the second sphere, which is at a large distance $((2 / \sqrt{3}-1) \Delta \simeq 0.15 \Delta)$ from the first sphere, is often important. In this case, $N_{b}=14$ and $q_{4}^{\mathrm{bcc}}=0.0363$ and $q_{6}^{\mathrm{bcc}}=0.510$.

Figure 1 shows the distributions of microparticles on the $q_{4}-q_{6}$ plane (calculated with $N_{b}=12$ ) at various crystallization stages of the confined Yukawa system: (a) the initial uncorrelated state of the ensemble of particles, (b) the intermediate glassy state, and (c) the almost complete crystallization of the system of microparticles. Thus, the presented distributions demonstrate a universal path that the confined Yukawa system of microparticles passes in the crystallization process on the $q_{4}-q_{6}$ plane. The crystallized Yukawa system consists primarily of hcp and fcc clusters (with a noticeable dominance of the hcp phase) and a rela- tively insignificant fraction of bcc clusters. The inset in Fig. 1c shows the typical time dependence of the parameter $q_{6}$ for a microparticle that becomes an fcc-like particle in the crystallization process, i.e., the kinetics of the formation of an fcc particle. The initial uncorrelated behavior of the microparticle (at $\left.q_{6}<0.4\right)$ is clearly seen (the particle often changes its nearest neighbors at this stage); then, the microparticle is transformed to an hcp-like particle (nearest neighbors remain unchanged, but only their conformation changes), and the transformation of the hcp particle to the fcc-like particle is finally observed.

A more detailed distribution of particles at various crystallization stages is shown in Fig. 2 for only one rotational invariant $q_{6}$. We point to the formation of a stable quasicrystalline (QC) phase (the corresponding peak in the presented distribution is between the hcp and fcc lattices with $q_{6} \simeq 0.52$ and the quasicrystalline lattice type is a torsion modification of the hep or fcc phase discovered for the first time in [19] and is presented in the inset in Fig. 2). The other inset in Fig. 2 shows the distribution of microparticles in the parameter $q_{4}$ calculated with $N_{b}=8$, which demonstrates the presence of the bcc phase in the crystallized Yukawa system.

The melting of the plasma crystal obtained in the molecular dynamics simulation was also calculated by the molecular dynamics simulations; in this calculations, the temperature of the Langevin thermostat, $T_{\mathrm{g}}$, was increased by a small value $\delta T_{\mathrm{g}} \ll T_{\mathrm{d}}$ and the subsequent calculation was terminated when the system of microparticles was transformed to the quasistable state with a new temperature of $T_{\mathrm{g}}+\delta T_{\mathrm{g}}$. Some features of the local order in the melting of the Yukawa system are shown in Figs. 3a and 3b. Figure 3a presents the initial stage of the melting of the plasma crystal (see Fig. 1c). 


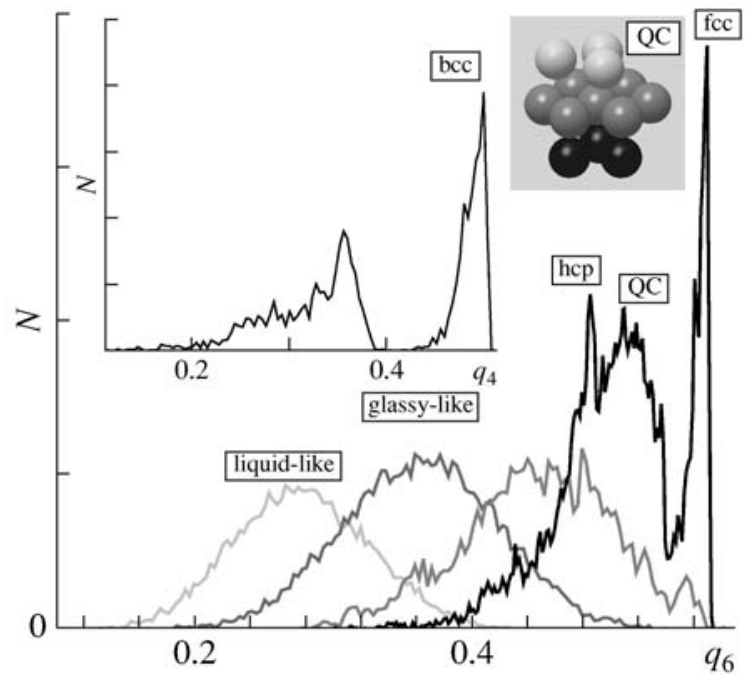

Fig. 2. (Color online) The particle distribution in the rotational invariant $q_{6}$ at various crystallization stages. The initial uncorrelated state, weakly correlated glassy state, beginning of crystallization, and the final state of the almost complete crystallization of the system of microparticles are shown. In the last case, the peak of the distribution between the hcp and fcc lattices $\left(q_{6} \simeq 0.52\right)$ corresponds to a stable quasicrystalline (QC) phase (the 12 nearest neighbors of the quasicrystalline phase are shown in the inset). The parameter $q_{6}$ is calculated with $N_{b}=12$. The inset also shows the distribution of microparticles in the parameter $q_{4}$ calculated with $N_{b}=8$. The peak of the distribution at $q_{4} \simeq 0.5$ indicates the presence of bcc clusters in the system.

With a small increase in the temperature of the thermostat $T_{\mathrm{g}}$ (or an decrease in the coupling parameter $\Gamma)$, the fcc and bcc clusters disappear almost completely (see Fig. 3b and inset in Fig. 3b). Thus, the density of the fcc/bcc particles in the system can be considered as a new important parameter sensitive to the beginning of the melting phase transition in the system. Another important feature of the crystallization/melting of such a system is the presence of the hysteresis on the $q_{4}-q_{6}$ plane, i.e., the melting and crystallization paths do not coincide with each other. In the process of melting, the parameter $q_{6}$ decreases, whereas the parameter $q_{4}$ does not noticeably change. On the contrary, both parameters $q_{6}$ and $q_{4}$ increase during the process of crystallization.

In this work, changes in the local order of the confined Yukawa system at various stages of crystallization and melting have been examined. It has been shown that the initial crystallization stage in the presence of the confinement in the form of a hard wall potential is accompanied by the formation of hcp-like clusters; a noticeable number of them are transformed to fcc-like clusters at a later crystallization stage. Moreover, fcc clusters are formed only from hcp clusters. The discussion of the kinetics of the formation of the fcc phase is
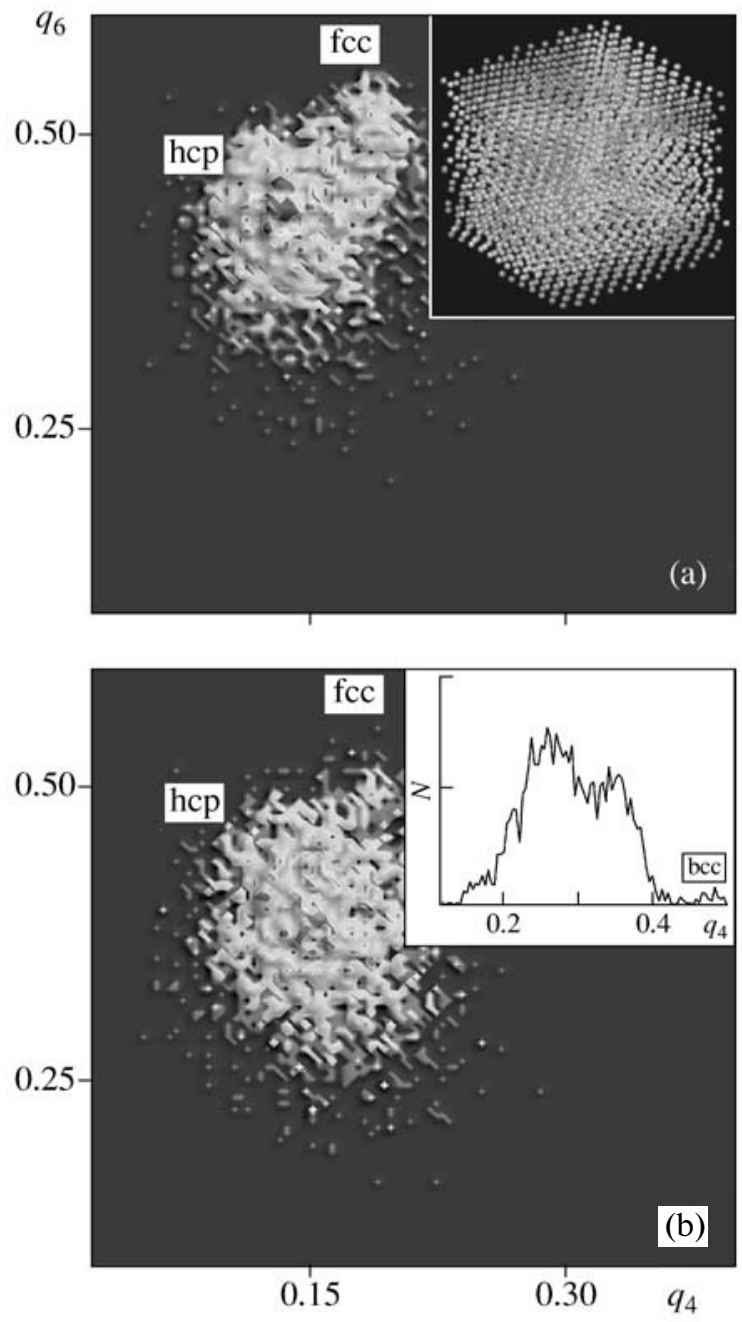

Fig. 3. (Color online) The distribution of microparticles on the $q_{4}-q_{6}$ rotational invariant plane, which demonstrates the structural properties of the confined Yukawa system at various melting stages: (a) the initial melting stage, i.e., the beginning of the disappearance of fcc clusters, and (b) the glassy state of the system of microparticles. The rotational invariants $q_{4}$ and $q_{6}$ are calculated with $N_{b}=12$. The inset in panel (a) shows the crystallized Yukawa system; the colors of microparticles (from green to red) are given according to $q_{6}$ values $(0.45-0.57$, respectively). The inset in panel (b) shows the distribution of microparticles in the parameter $q_{4}$ calculated with $N_{b}=8$, indicating an almost complete disappearance of the bcc phase in the system.

beyond the scope of this work and will be given in the next publications. A few bcc clusters were also observed in the crystallized Yukawa system. With the beginning of the melting of the plasma crystal, fcc and bcc phases disappear first with a small decrease in the coupling parameter $\Gamma$. This means that the density of the $\mathrm{fcc} / \mathrm{bcc}$ clusters can be used as an alternative important indicator of the phase transition (melting) in the confined Yukawa system. 


\section{REFERENCES}

1. P. K. Shukla and A. A. Mamun, Introduction to Dusty Plasmas Physics (Inst. of Physics, Bristol, 2002).

2. V. E. Fortov et al., Phys. Rep. 421, 1 (2005).

3. M. Horanyi et al., Rev. Geophys. 42, RG4002 (2004).

4. B. A. Klumov, S. V. Vladimirov, and G. E. Morfill, JETP Lett. 85, 478 (2007).

5. B. A. Klumov, S. I. Popel, and R. Bingham, JETP Lett. 72, 364 (2000); B. A. Klumov, S. I. Popel, and G. E. Morfill, JETP 100, 152 (2005); B. A. Klumov, S. V. Vladimirov, and G. E. Morfill, JETP Lett. 82, 632 (2005).

6. V. E. Fortov et al., Usp. Fiz. Nauk 166, 391 (1996) [Phys. Usp. 166, 363 (1996)]; B. A. Klumov et al., Phys. Usp. 48, 733 (2005).

7. G. Morfill et al., Contirb. Plasma Phys. 44, 450 (2004); G. Morfill et al., Phys. Scripta T 107, 59 (2004).

8. M. O. Robbins et al., J. Chem. Phys. 88, 3286 (1988).

9. G. Morfill et al., Phys. Rev. Lett. 92, 175004 (2004).
10. M. Rubin-Zuzic et al., Nature Phys. 2, 181 (2006); B. A. Klumov, M. Rubin-Zuzic, and G. E. Morfill, JETP Lett. 84, 542 (2006).

11. M. Zuzic et al., Phys. Rev. Lett. 85, 4064 (2000).

12. O. Arp et al., Phys. Rev. Lett. 93, 165004 (2004).

13. S. Mitic et al., Phys. Rev. Lett. 101, 125002 (2008).

14. J. H. Chu and L.I., Phys. Rev. Lett. 72, 4009 (1994).

15. H. Thomas et al., Phys. Rev. Lett. 73, 652 (1994).

16. H. Ikezi, Phys. Fluids 29, 1764 (1986).

17. U. Konopka, G. Morfill, and L. Ratke, Phys. Rev. Lett. 84, 891 (2000).

18. P. J. Steinhardt, D. R. Nelson, and M. Ronchetti, Phys. Rev. B 28, 784 (1983).

19. B. A. Klumov and G. E. Morfill, JETP Lett. 85, 498 (2007); JETP Lett. 87, 409 (2008); JETP 107, 908 (2008).

20. S. Auer and D. Frenkel, J. Chem. Phys. 120, 3015 (2004).

Translated by R. Tyapaev 